\title{
Constraint to the Satisfactory Conditions for R\&D Endogenous Growth Model
}

\author{
Cai Defa ${ }^{\mathrm{a}}$, Ma Wendi ${ }^{\mathrm{b}}$ \\ Finance and Public Management College, Harbin University of Commerce, P.R. China, 150028 \\ aabcd04754@126.com, bmawendi619@163.com
}

Keywords: Resource Constraint; R\&D; Endogenous Growth model

\begin{abstract}
Low-Carbon Resources include low-consumption energy like renewable resources, new energy, clean power, etc. From the perspective of fiscal policy, to study and develop R\&D will affect the progress of technology directly, and to develop Low-Carbon Economy needs the innovation of Low-Carbon technology. Thus, the government's R\&D investment is crucial to developing Low-Carbon technology and Low-Carbon Economy. This thesis combines the endogenous growth theory with the sustainability of Low-Carbon Resources, and uses the R\&D endogenous growth model to give one under the constraint of Low-Carbon resources. It comes to a relevant conclusion, that is, assuming that the society requires a sustainable consumption in consuming the rest of exhaustible resource, the labor investment of R\&D sectors should meet the basic conditions.
\end{abstract}

\section{Introduction}

With the characteristics of low energy consumption, low pollution, low emission based on economic model, low carbon economy is a trend of world economic development. Low carbon economy is a great progress of human society after the agricultural civilization, industrial civilization. The essence of low-carbon economy is the high energy efficiency and clean energy structure, the core is a fundamental change of energy technology innovation, system innovation and the concept of human survival and development. Based on the theory of low carbon economy foundation, Construction and validation of endogenous growth model through inside, draw if society in the consumption of the exhaustible resource stock period required to maintain the sustainable consumption, R\&D Department of labor input conditions should be met.

The endogenous theory was established in the 1980s, when the economists were studying the growth rate under complete competition, examining that the decisive factor of the economy's sustainable growth was the progress of the endogenous technology, that is, with the continuous advancement of which, the economy can keep sustainable growing without external mechanisms. At the beginning of the 1990s, such representatives of growth theorists like P.Romer of New York University’s Stern School of Business (1986. 1987. 1990), American economist Lucas(1985), Robert Barro, he vice president of American Economist Association began to study the economic growth under the condition of monopolistic competition, and put forward the endogenous growth model, which modified the neo-classical economic growth production function. It took the technology's progress, human capital, and scientific study and development as the virables of economic growth, putting forward the endogenous theory, and in which gave 3 reasons of the economic growth stated: the first was to acquire new knowledge, including innovation, technology's growth and human capital's accumulation, ect.; The second was stimulating new knowledge applied to production; The third was to offer new resources of using new knowledge, including humanpower, capital, imports and ect. Among whom, Barro(1990) introduced the public sections into the AK production function of constant returns to scale to found a government-centered endogenous growth model and get the optimal scale of Financial Expenditure. 


\section{The Construction of the Model}

In order to use Low-Carbon Resources properly, the relationship between two aspects needs well handled. On the one hand, Low-Carbon resources should be used properly, that is, restricting Low-Carbon resources within the threshold value,or else it would lead to an irreversible destruction to its regenerative ability; On the other hand, it should consider about two sectors, that is, the producing sector and R\&D sector, the labor force could flow freely between them.

This thesis has inserted the Low-Carbon Resources into the production function to establish a simple endogenous growth model. R\&D section innovates new product, and the amount of which represents new technology level, to put the equation of technology's variation as:

$\bar{A}=\zeta A \cdot L_{A}$

Among which, A is producing section's technology level; $\bar{A}$ is the rate of technology change; ${ }^{L_{A}}$ is the labor force investment of $\mathrm{R} \& \mathrm{D}$ section, $\zeta$ is the parameter of productivity. The production function of producing section is: $Y=A \cdot F\left(K, L_{M}, R\right)$

Among which, $\mathrm{K}$ is the capital investment of production sector; $\mathrm{R}$ is the Low-Carbon elements; $L_{M}$ is the labor force investment. Assuming that: the aggregate labor force is constant $1\left(\mathrm{~L}=L_{A}{ }^{+}\right.$ $L_{M}$ ), $L_{M}$ and $L_{A}$ are the labor which put into the producing and R\&D sectors.

In order to prevent the stock of total resources being used up in limited time, ensuring the sustainable use of Low-Carbon Resources, it needs to reduce the investment of Low-Carbon Resources gradually, and put the production function of the production's producing section as:

$$
Y=A K^{\alpha} L_{M}^{\beta} R^{\lambda}
$$

Among which, $0<\alpha, \beta, \gamma<1$. The equation of capital's variation is:

$$
\bar{K}=A K^{\alpha} L_{M}^{\beta} R^{\lambda}-c L-\eta K
$$

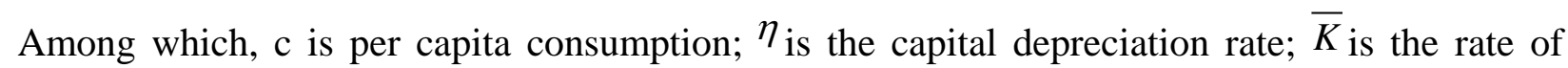
capital variation.

Assuming that, in order to meet the goal of Low-Carbon benefit, under the condition of Low-Carbon Resources' continuous usage, the government may choose $L_{A}$ 's time usage maximization of consuming and R\&D sections, which is: $\max U=\max \int_{0} \exp (-\rho t) \ln (c l) d t$

Among which, $P$ is discount $\operatorname{rate}(\rho>0)$.

\section{The Solution of the Model}

The study of growth shows that, most countries develop steadily in a long term. Thus, we suppose that, long-term growth has a steady feature, and the growth rate of per capita variable is constant in the long-term development. Using the pontryagin's maximum principle to deal with dynamic optimization, the Hamiltion function can be put as:

$$
H=\exp (-\rho t) \ln (c l)+\mu \exp (-\rho t)\left[\zeta A\left(L-L_{M}\right)\right]+\lambda \exp (-\rho t)\left[A K^{\alpha} L_{M}{ }^{\beta} R^{\lambda}-c L-\eta K\right]
$$

Among which, $\mu$ 、 $\lambda$ are shadow price of technology and capital respectively . From the first order condition and derivation we can get:

$$
\lambda=c^{-1}
$$




$$
\begin{aligned}
& \lambda \frac{\partial Y}{\partial L_{M}}=\mu A \zeta \\
& -\bar{\mu}+\rho \mu=\mu \zeta\left(L-L_{M}\right)+\lambda \frac{\partial Y}{\partial A} \\
& -\bar{\lambda}+\lambda \rho=\lambda \frac{\partial Y}{\partial \mathrm{K}}-\lambda \eta
\end{aligned}
$$

Plugging (5) into (6), we can get:

$$
\frac{\bar{\mu}}{\mu}=\rho-\zeta \mathrm{L}_{\mathrm{A}}-\frac{\zeta}{\beta}\left(L-L_{\mathrm{A}}\right)
$$

Plugging (5) into (7), we can get:

$$
\frac{\bar{\lambda}}{\lambda}=\rho+\eta-\frac{\alpha \mu \zeta A L_{M}}{\beta \lambda K}
$$

Assuming that the variable is $\mathrm{x}$, the growth rate of $\mathrm{x}$ is $r_{x}$, we can get: $r_{c}=\frac{\bar{c}}{C}, r_{k}=\frac{\bar{K}}{K}, r_{R}=\frac{\bar{\lambda}}{\lambda}, r_{\mu}=\frac{\bar{\mu}}{\mu}, r_{A}=\frac{\bar{A}}{A}$

From (4), we can get:

$$
\frac{\bar{\lambda}}{\lambda}=-\frac{\bar{c}}{c}=-r_{c}
$$

Under the condition of steady growth, each variable's growth rate is constant, and $L_{M}$ is constant, so, from form (10) we can get the right of form (9)is constant. Then, we comes to the conclusion: $\frac{\alpha \mu \zeta A L_{M}}{\beta \lambda K}=$ constant,the derivative of which is:

$$
r_{\mu}+r_{A}=r_{k}+r_{\lambda}
$$

From form (3): ${ }^{r_{k}}=\frac{\bar{K}}{K}=A \frac{K^{\alpha} L_{m}{ }^{\beta} R^{\lambda}}{K}-\frac{c L}{K}-\eta$ is constant, and c/K is constant, we can get :

$$
r_{k}=r_{c}
$$

From $A \frac{K^{\alpha} L_{M}{ }^{\beta} R^{\lambda}}{K}=$ constant, to get derivative of both sides, and plug form (1) into $r_{A}=\zeta L_{A}$, we can get:

$$
\zeta L_{A}+(\alpha-1) r_{k}+\gamma r_{R}=0
$$

From form (1)(2)(5), we can get: ${ }^{L_{M}}=\left[\frac{\mu \zeta}{\lambda \beta K^{\alpha} R^{\gamma}}\right]^{1 /(\beta-1)}$, and then get:

$$
\frac{\bar{A}}{A}=\zeta L-\zeta\left[\frac{\mu \zeta}{\lambda \beta K^{\alpha} R^{\gamma}}\right]^{1 /(\beta-1)}
$$

Having the derivative of time, we can get:

$$
r_{\mu}=r_{\lambda}+\alpha r_{k}+\gamma r_{R}
$$

Through the solution of equation (1)、(8)、(11)、(12)、(14)and(15),we can get:

$$
L_{A}=\frac{(\zeta L-\rho \beta)}{\zeta}
$$


The sustainable growth of economy is one of the necessary conditions of sustainable development. Thus, under the condition of maintaining positive growth of per capita consumption, per capita consumption keeps positive growth, and under this condition, from(12)、(13)and(16), we can get: $r_{c}=r_{k}=\frac{1}{1-\alpha}\left[\gamma_{r_{R}}+L \zeta-\rho \beta\right]>0$

From $0<\alpha<1$, we can get:

$$
\gamma\left(-r_{R}\right)<L \zeta-\rho \beta
$$

The sustainable use of low-carbon resources requires $r_{R<0}$, that is $r_{R=-} r_{R}$, so, form (17) is written as:

$$
r_{R}<\frac{1}{\gamma}(L \zeta-\rho \beta)
$$

Thus, to satisfy the sustainable growth of economy and sustainable use of low-carbon resources, it needs the absolute input growth rate of the low-carbon resources in the producing process should less than $(L \zeta-\rho \beta) / \gamma$.

Then from (1) and (18), we can get: $L_{\mathrm{A}}>\frac{\gamma}{\zeta} r_{R}$

This form is supposing that society requires to maintain sustainable consumption during consuming the stock of low-carbon resources, and the labor force investment of R\&D shoud meet the needs.

\section{Conclusion}

Using the R\&D endogenous growth model, to establish a simple one under the constraint of low-carbon resources, and then combine sustainable development of low-carbon resources with endogenous growth theory, we can comes to the conclusion: through increasing section R\&D's labor force to push forward technology's progress, supposing that society requires to maintain sustainable consumption in consuming the stock of low-carbon resources for long, the conditions of section R\&D's labor force investment should meet is: ${ }^{L_{A}>\frac{\gamma}{\zeta} r_{R}}$.Thus, in fiscal aspect, supposing that society requires to maintain sustainable consumption in consuming the stock of low-carbon resources for long, For a long time R\&D sector should meet the need.

\section{Acknowledgement}

Foundation Project: The national social science fund project(17BJY177); The project for philosophy and social science research base of Heilongjiang Province(16JYD19)

\section{References}

[1] Li Sun.Development and evolution of the endogenous growth theory [J].Modern economic information, 2012(10):272

[2] Yutong Li. Based on 3 e coordination degree analysis path of low carbon economy development of heilongjiang province [J].Journal of qiushi journal 2013(1):73-79

[3] Zhu Yibin, Liu Jiashun. Research on Connotation of Low Carbon Economy [J].Energy Policy. 2013(6):198-201

[4] Ashok Kumar Jain.Low carbon city, policy, planning and practice [M].2001, 375-379 
[5] G.Y.Zhuang.The Way of China's Low-Carbon Economy Development and Analysis of its Potential [J].International Technical and Economic Research 2005(7), 79-87

[6] Great Britain.The Economics of Climate Change: the Stern review [J].Cambridge 2016(2), 15-16

[7] R. Sathiendrakuma. Green house emission reduction and sustainable development [M]. International Journal of social Economic IEEE Network.2003: 2033-2048

Cai Defa(1966-):professor,Ph.D,Research Direction:Taxation Theory and Regulation Design;Ma Wendi(1996-),Woman,Public Finance Master Graduate Student of Finance and Public Management Institute. 